\title{
Genomweite Assoziationsstudien zu Adipositas und was wir daraus lernen
}

\author{
Florian Kronenberg · Bernhard Paulweber · Claudia Lamina
}

Eingegangen: 3. November 2015 / Angenommen: 30. Dezember 2015 / Online publiziert: 21. Januar 2016

(C) Die Autor(en) 2016. Dieser Artikel ist auf Springerlink.com mit Open Access verfügbar.

\begin{abstract}
Zusammenfassung Die Einführung genomweiter Assoziationsstudien (GWAS) führte $\mathrm{zu}$ einem sprunghaften Anstieg der Anzahl von Genen, die mit Adipositas und zusammenhängenden Phänotypen wie BodyMass-Index, Taillenumfang und Taille-Hüft-Quotient assoziiert sind. Dabei ist die Suche nach Genen, die für Übergewicht prädisponieren erst am Anfang, denn der Großteil des geschätzten vererbbaren Anteils von Übergewicht ist noch ungeklärt. Jedes einzelne der 97 bisher bekannten Gene für Body-Mass-Index und 49 Gene für Taille-Hüft-Quotient erklärt nur einen sehr kleinen Anteil der Varianz dieser Phänotypen. Geschlechtsspezifische Unterschiede kennen wir hauptsächlich nur für den Phänotyp Taille-Hüft-Quotient und ca. zwei Fünftel der dafür bekannten Genvarianten weist vor allem bei Frauen einen Effekt auf und keinen oder einen deutlich geringeren Effekt bei Männern. Die funktionelle Charakterisierung dieser Gene wird lange Zeit in Anspruch nehmen. Ob und wie schnell sich aus den Ergebnissen therapeutische Konsequenzen ergeben, lässt sich schwer abschätzen. Das Verständnis involvierter Regelkreise ist aber unabdingbare Voraussetzung für die Entwicklung neuer Therapien.
\end{abstract}

\footnotetext{
Univ.-Prof. Dr. F. Kronenberg $(\bowtie) \cdot$ C. Lamina

Division für Genetische Epidemiologie, Department für

Medizinische Genetik, Molekulare and Klinische Pharmakologie,

Medizinische Universität Innsbruck,

Schöpfstr. 41,

6020 Innsbruck, Österreich

E-Mail: Florian.Kronenberg@i-med.ac.at

B. Paulweber

Universitätsklinik für Innere Medizin I der Paracelsus

Medizinischen Privatuniversität Salzburg, St. Johanns-Spital,

Müllner Hauptstraße 48,

5020 Salzburg, Österreich
}

Schlüsselwörter Genetische Variabilität .

Übergewicht · Body-Mass-Index · Taille-Hüft-Quotient · Genomweite Assoziationsstudie $\cdot$ Heritabilität

Genomwide association studies on obesity: what can we learn from these studies

Summary The introduction of genome-wide association studies resulted in a tremendous increase in the number of genes associated with obesity and related phenotypes (BMI, waist and waist-hip-ratio). Despite this enormous gain in knowledge the search for genes is only started since only a small fraction of the heritability of these phenotypes is explained yet: each single gene of the 97 hitherto known BMI-associated genes and 49 waist-hipratio-associated genes explains only a tiny fraction of the variance of these phenotypes. Sex-specific differences are mainly known for waist-hip-ratio and $\sim 40 \%$ of the genes showed only an effect in women but no or a markedly smaller effect in men. The functional characterization of the identified genes will take a lot of time. It is unclear whether and how fast the findings will result in therapeutic consequences. It is of utmost importance that we understand the involved mechanisms before new therapeutic strategies can be developed.

Keywords Genetic variability · Obesity · Body-Mass-Index · Waist-hip-ratio ·

Genome-wide association study $\cdot$ Heritability

\section{Warum sollte Genetik unser Körpergewicht überhaupt beeinflussen?}

Es besteht kein Zweifel, dass die Häufigkeit der Adipositas in der Bevölkerung über die letzten Jahrzehnte dramatisch zugenommen hat. Diese Zunahme 
ist allerdings nicht nur durch das Überangebot und die leichte Zugänglichkeit zu Nahrung in unseren geographischen Breiten bedingt, sondern basiert zum Teil auch auf einer entsprechenden genetischen Ausstattung, die jedes Individuum $\mathrm{zu}$ einem unterschiedlichen Umgang mit Nahrung und der Verwertung von Nahrung „prädisponiert“. Kritiker werfen an dieser Stelle sehr häufig ein, warum Genetik hier eine wichtige Rolle spielen sollte, wo doch der Anstieg der Prävalenz der Adipositas in den letzten Jahrzehnten zu beobachten war, vor allem aber die Genetik auf Populationsebene als relativ „stabil“ auf die kurze Zeitspanne gesehen werden kann. Oder mit anderen Wort: Es ist sicherlich nicht zu erwarten, dass sich in den letzten Jahrzehnten genetische Mutationen entwickelt haben, die zu Adipositas prädisponieren. In diesem Zeitraum hat aber auch z. B. die durchschnittliche Körpergröße zugenommen. Trotzdem würde kaum jemand die genetische Komponente bezweifeln, die der Körpergröße zugrunde liegt. Man nimmt an, dass es über Millionen von Jahren zu einer Akkumulation von Allelen (Genvarianten) gekommen ist, die jede für sich einen kleinen Beitrag zur Adipositas leistet, der aber unter „normalen“ Ernährungsbedingungen schwer phänotypisch fassbar ist, wenn man diese Genvarianten einzeln betrachtet. Durch die deutliche Veränderung hin zu einer nahrungsreichen und bewegungsarmen Umgebung erfahren diese Genvarianten eine „sichtbare" Bedeutung, indem sie zu einem additiven und leichter messbaren Effekt führen. Man kann dies auch als klassische Gen-Umwelt-Interaktionen bezeichnen. Dabei wurden in den letzten Jahrzehnten mehrere verschiedene Blickwinkel der „thrifty gene“ Hypothese diskutiert: einerseits könnten evolutionsgeschichtlich Träger von Mutationen, die zu einer effektiveren Fetteinlagerung führen, in Zeiten von Hunger von Natur aus bevorteilt sein. Diese Mutationen hätten sich dann über Jahrtausende im Sinne einer positiven Selektion durchgesetzt [1]. Andererseits geht man von genetischem Drift aus, unter dem zufällige Mutationen entstehen, die aber über die Jahrtausende keinem positiven Selektionsdruck unterworfen waren, unter heutigen Umweltbedingungen aber zu einem entsprechenden Gewichtsanstieg führen, wenn man zufällig zahlreiche dieser Mutationen von seinen Vorfahren geerbt hat [2]. Gengruppen, die entsprechende Beiträge leisten dürften, sind aus den Bereichen Appetit-Regulation, Metabolismus und Thermogenese, Prädisposition zu körperlicher Aktivität, Kapazität der Fetteinlagerung in Adipozyten sowie Lipidoxidation [3]. Die genauere Betrachtungsweise der zugrundeliegenden Überlegungen wurden kürzlich ausführlich von Jonathan C.K. Wells dargelegt [4].

\section{Wie ausgeprägt ist die genetische Komponente bei der Adipositas?}

Aus Familienstudien sowie Zwillings- und Adoptionsstudien nimmt man an, dass die genetische Komponente für Adipositas sehr ausgeprägt ist. Die geschätzten
Heritabilitäten (= Maßzahl für vererbbaren Anteil eines Phänotyps) liegen zwischen 50 und $80 \%$ [5-7], wobei diese Daten in der Regel immer in industrialisierten Ländern erhoben worden sind. Gerade in Anbetracht der oben angesprochenen ,thrifty gene“ Hypothese wäre es interessant, solche Berechnungen aus Ländern mit geringem Anteil an Überernährung zu kennen oder auch in Populationen, die in den letzten Jahrzehnten einen recht deutlichen Übergang zu einer sogenannten „western-type diet" mit deutlichem Anstieg der Adipositas vollzogen haben mit der Untersuchung der Heritabilität vor und nach dem Übergang.

Diese hohe Heritabilität und die leichte Zugänglichkeit zu individuellen Daten in Form des Body Mass Index (BMI) oder anderer Parameter wie Taillenumfang waren natürlich einladend für großangelegte Studien, die zum Ziel haben, Gene zu finden, die diese Phänotypen beeinflussen. Es gibt einzelne monogenetische Erkrankungen oder Syndrome, die unter anderem zu Adipositas führen $[8,9]$. Diese sind aber nur für einen kleinen Anteil der Adipositas-Fälle verantwortlich. Die Mehrzahl der Fälle basiert auf einem komplexen Zusammenspiel von vielen genetischen Varianten und anderen Faktoren, wir z. B. Ernährung und Bewegung. So soll im Rest dieses Überblicks vor allem auf gefundene Genregionen eingegangen werden, die auf der Populationsebene im Sinne von komplexen Phänotypen eine Rolle spielen.

\section{Genomweite Assoziationsstudien zu Parametern des Übergewichtes}

Genomweite Assoziationsstudien (GWAS) haben die Suche nach Genen, die zu komplexen Phänotypen wie jenen der Gewichtsregulation beitragen, revolutioniert. Vor Einführung der GWAS wurden hauptsächlich Kandidatengenstudien durchgeführt, in denen gezielt aufgrund von biochemischen oder physiologischen Überlegungen Gene ausgewählt wurden. Einzelne Genvarianten (in der Regel Single-Nucleotide Polymorphisms, SNPs) dieses Gens wurden dann in einer entsprechenden populationsbasierten Studie oder einer Fall-Kontrollstudie untersucht (Abb. 1). Auf dem Prüfstand steht dabei jeweils die Hypothese, ob es eine Assoziation zwischen diesen Genvarianten und dem Phänotyp gibt. Dabei unterscheiden wir einen qualitativen und einen quantitativen Zugang (Abb. 2). Beim qualitativen Zugang wird untersucht, ob bestimmte Genvarianten gehäuft bei bestimmten Krankheiten wie Diabetes mellitus, koronarer Herzkrankheit, Adipositas usw. auftreten. Beim quantitativen Zugang wird untersucht, ob die Träger von bestimmten Allelen oder Genotypen einen höheren BMI, einen höheren TailleHüft-Quotient (Waist-Hip-Ratio=WHR), oder höhere Laborparameter aufweisen, um nur wenige Phänotypen zu nennen. Die Anzahl der entdeckten Genotyp-Phänotyp-Beziehungen für komplexe Erkrankungen hat sich seit Einführung der GWAS vor weniger als 10 Jahren mehr als verzehnfacht [10]. Dies liegt daran, dass man bei GWAS einen „unvoreingenommenen“ Zugang wählt, indem man 
Kandidatengen-Untersuchung

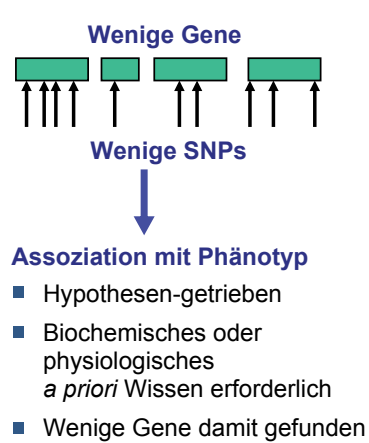

Genomweite Assoziationsstudie GWAS

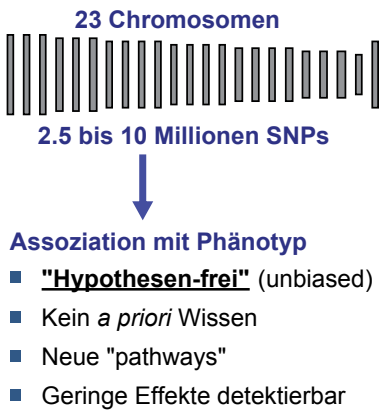

Abb. 1 Prinzip von Kandidatengen-Untersuchungen und genomweiten Assoziationsstudien (GWAS)

das Genom systematisch nach Genvarianten durchsucht, die mit dem Phänotyp von Interesse in Assoziation stehen. Man versucht dabei möglichst alle Gene zu berücksichtigen, indem man zumindest die relativ häufigen Genvarianten mit einer Häufigkeit von mehr als $1 \%$ in der Bevölkerung in der Analyse berücksichtigt. In der Regel werden dabei ca. 2,5-10 Mio. Genvarianten untersucht (Abb. 1). Aufgrund der vielen durchgeführten statistischen Tests und der Gefahr von falsch-positiven Ergebnissen, braucht man hierzu meist sehr große Fallzahlen, die oft in die zehntausende und mehr gehen, da man für die Anzahl der statistischen Tests (= Anzahl der untersuchten SNPs) korrigieren muss [11]. Dies führt dazu, dass bei diesen Analysen erst Assoziationen mit einem statistischen p-Wert kleiner als $5^{*} 10^{-8}$ als genomweit signifikant angesehen werden und für die weitere Nachverfolgung berücksichtigt werden. Der Hauptvorteil dieser Methode ist allerdings, dass man damit auch Assoziationen mit bisher nicht bekannten Genen finden kann, auf die man bei einer hypothesen-getriebenen Genauswahl kaum kommen würde.

Im Folgenden soll über die wichtigsten Ergebnisse dieser GWAS in Bezug auf Adipositas-assoziierte Phänotypen wie Body-Mass-Index, WHR, Taillenumfang und assoziierte Phänotypen berichtet werden. Soweit es dazu bereits geschlechtsspezifische Daten gibt, werden diese ebenfalls dargestellt. Nicht eingegangen wird auf die zahlreichen oftmals als Kandidatengen-Studien durchgeführten Untersuchungen, die sehr häufig einer Bestätigung harren.

\section{GWAS Ergebnisse bezüglich BMI}

Die beiden größten bisher durchgeführte genomweiten Metaanalysen zu BMI stammt vom GIANT-Konsortium (http://www.broadinstitute.org/collaboration/giant/ index.php/Main_Page), welches insgesamt Daten von fast 340.000 Personen aus 125 verschiedenen Kohorten nutzte [12, 13]. Dabei konnten 97 Genorte identifiziert werden, von denen wiederum 56 noch nicht vorher mit Adipositas in Verbindung gebracht worden waren und

Prinzip von genetischen Assoziationsstudien

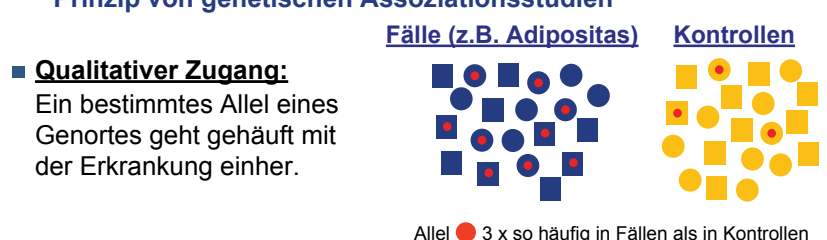

- Quantitativer Zugang:

Die einzelnen Gruppen von Allel-

Trägern haben unterschiedliche

Höhen eines kontinuierlichen

Messwertes (z.B. BMI,

Cholesterin, ...)

Allel $3 x$ so häufig in Fällen als in Kontrollen

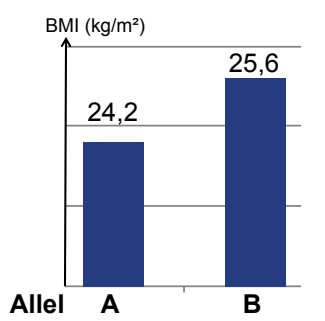

Abb. 2 Prinzip von genetischen Assoziationsstudien

somit neu waren. Insgesamt erklären diese 97 Genorte nur 2,7\% der phänotypischen Varianz vom BMI. Nur ganz wenige einzelne Gene erklären mehr als $0,1 \%$ der Varianz [12]. Man geht davon aus, dass noch viele Hunderte SNPs zum Phänotyp BMI beitragen und noch der Entdeckung harren. Dieses geringe Ausmaß erklärter Varianz rief natürlich Kritiker auf den Plan, die die Frage in den Raum stellten, was das Wissen um Genvarianten nützen soll, welche deutlich unter $1 \%$ der Varianz erklären. Dem kann entgegen gehalten werden, dass ein Gen, dessen Varianten eventuell nur sehr geringe Effekte in Assoziationsstudien zeigen, trotzdem eine wichtige Rolle für den Phänotyp spielen kann und zwar in viel größerem Ausmaß, wie es die GWAS Ergebnisse vermuten lassen. Als Beispiel sei die HMG-CoA Reduktase angeführt, welches im Cholesterinstoffwechsel als eines der Schlüsselenzyme gilt. Häufige genetische Varianten in diesem Gen erklären bei weitem weniger als $1 \%$ der Cholesterinkonzentrationen. Trotzdem ist es unbestritten bereits vor langer Zeit zu einem der besten Ansatzpunkte für cholesterinsenkende Therapie mittels Statinen geworden. Mittlerweile sind mehrere andere Gene, die durch GWAS identifiziert worden sind, $\mathrm{zu}$ interessanten Kandidaten für Medikamentenentwicklungen, sogenannten „Drug-Targets“, geworden. In der GWAS zu BMI wurden z. B. Gene aus dem GlutamatRezeptor Pathway identifiziert, die durch Nahrungsaufnahme bzw. Fasten gesteuert werden. In diesem Pathway soll auch Topiramate eingreifen, welches in zwei von der amerikanischen Food and Drug Administration (FDA) zugelassenen Medikamenten zur Gewichtsreduktion zum Einsatz kommt [13]. In den GWAS Ergebnissen verstecken sich also vermutlich noch weitere potentiell interessante Kandidaten für Medikamentenentwicklungen. Man wird sehen, welche dieser neuen Ansatzpunkte in Jahren therapeutischen Einzug in die Klinik finden werden.

Die Autoren dieser großen BMI-GWAS betonen, dass eine überproportional hohe Anzahl der gefundenen Genregionen bei näherer Betrachtung mit der neuronalen Regulation von Appetit und Energie-Homeostase 
in Zusammenhang stehen (z. B. MC4R, BDNF, SH2B1 oder $P O M C$ ). Es sind auch bereits seltene Varianten in diesen Genen bekannt, die zu sehr schweren monogenen Formen von Übergewicht führen [12, 13]. Die Entdeckung von häufigen Varianten in diesen Genen, die mit geringfügigen Änderungen des BMI einhergehen, erweitert die Sichtweise auf ein sehr weites Spektrum von Genotyp-Phänotyp Beziehungen sogar innerhalb desselben Genes. Neben diesen zentral-neuronal exprimierten Genen haben sich auch Genvarianten gefunden, die mit der Insulinsekretion und Insulinwirkung, dem Energiestoffwechsel, dem Lipidstoffwechsel und der Adipogenese in Zusammenhang stehen [13].

In diesen Studien konnte auch sehr schön der additive Effekt der Risiko-Allele gezeigt werden [12, 13]. Bei 97 Genvarianten, die mit BMI assoziiert sind, können minimal 0 und maximal 194 Risikoallele auftreten. Diese Extreme sind allerdings höchst selten. Der Großteil der Bevölkerung trägt zwischen 85 und 98 Risikoallele. Es gibt allerdings mit $1.16 \%$ einen relativ kleinen Anteil an Menschen, die mit $<78$ Allelen eine sehr geringe Anzahl an Risikoallelen trägt sowie eine ähnlich kleine Gruppe mit 1,78\%, die mit > 104 Allelen eine sehr hohe Anzahl an Risikoallelen trägt. Diese beiden Gruppen unterscheiden sich in ihrem BMI um 3,3 kg/m ${ }^{2}$, was im Schnitt ungefähr 8-11 kg Körpergewicht bei Personen mit 160-180 cm Körpergröße entspricht. Dieses Ausmaß muss bereits als sehr relevant für die Entwicklung von metabolischen Erkrankungen betrachtet werden. Über den gesamten Wertebereich der Risikoallele ließ sich ein sehr klarer linearer Zusammenhang zeigen: Je höher die Anzahl der Risikoallele war, umso höher war der BMI. Auch wenn dieser Zusammenhang statistisch signifikant und die Unterschiede zwischen den Extremgruppen bereits klinisch relevant sind, kann ein derartiger genetischer Risikoscore (noch) nicht für individuelle Risikovorhersagen genutzt werden. Dafür ist ein zu großer Teil der BMI-Variabilität noch nicht geklärt.

Nur für zwei der 97 Genorte (SEC16B und ZFP64) konnten geschlechtsspezifische Unterschiede gefunden werden, wobei die Effekte bei Frauen stärker als bei Männern waren [13].

\section{GWAS Ergebnisse bezüglich Phänotypen der zentralen Fettverteilung}

Generell besteht eine klare Beziehung zwischen einer erhöhten Körperfettmenge und dem Risiko für Typ-2 Diabetes mellitus und verwandte Komorbiditäten, wie nicht-alkoholische Fettlebererkrankung, kardiovaskuläre Erkrankungen und verschiedene Neoplasien [14]. Eine wesentlich stärkere Rolle als die Gesamtkörperfettmenge spielt für diese Zusammenhänge jedoch das Fettverteilungsmuster. Eine erhöhte Fettansammlung in den viszeralen Fettdepots („viszerale“, „omentale“ oder „intra-abdominelle Adipositas“) weist so eine deutlich stärkere Assoziation mit den genannten Erkrankungen auf als eine Vermehrung von subkutanem Fett. Die mit viszeraler Adipositas assoziierte Insulinresistenz dürfte eine zentrale Rolle in der Pathophysiologie dieser Störungen spielen $[15,16]$.

Eine hohe Waist-Hip-Ratio (WHR, Taille-Hüft-Quotient) weist auf eine erhöhte intra-abdominale Fettablagerung hin, die mit einem erhöhten Risiko für Typ-2 Diabetes mellitus und kardiovaskulären Erkrankungen assoziiert ist $[17,18]$. In zwei durch das GIANT Konsortium durchgeführten Meta-Analysen zu WHR wurden Daten von knapp 225.000 Personen aus mehr als 100 Studien ausgewertet $[19,20]$. In der ersten Analyse waren 14 Genorte identifiziert worden, wobei 13 davon erstmals berichtet worden waren [19]. Die zweite dieser Studien identifizierte wiederum eine beträchtliche Anzahl von neuen Genen, sodass insgesamt 49 Genorten für WHR bekannt wurden [20]. Diese Studien weisen zwei Besonderheiten auf: 1) WHR wurde auf BMI adjustiert, um vor allem Gene zu finden, die unabhängig vom BMI einen Effekt auf die WHR und damit die Fettverteilung an sich haben; und 2) es wurde in dieser Studie systematisch auf Geschlechtsunterschiede untersucht. Dabei hat sich ein sehr deutlicher geschlechtlicher Dimorphismus gezeigt: Bei 19 der 49 Genorte waren die Geneffekte bei Frauen signifikant stärker ausgeprägt als bei Männern; nur ein Genort zeigte bei Männer signifikant stärkere Effekt als bei Frauen (Abb. 3). Aus diesen beiden Studien wurde geschlossen, dass es mehrere Gene gibt, die die Körperfettverteilung unabhängig vom Körpergewicht bzw. BMI beeinflussen und zwar in großem Maße abhängig vom Geschlecht. Dies weist auf eine deutliche Gen-Geschlechts-Interaktion hin [19, 20]. Die gefundenen Gene für WHR sind gehäuft im Fettgewebe exprimiert bzw. stehen mit regulatorischen Elementen in den Adipozyten in Zusammenhang. Pathyway-Analysen weisen auf eine Rolle in der Adipogenese, Angiogenese sowie die transkriptionelle Regulation und Insulinresistenz hin, die wiederum die Fettverteilung beeinflussen [20].

Ein weiterer Parameter für die zentrale Fettverteilung ist der Taillenumfang. Auch hiermit wurden GWAS durchgeführt: Chambers et al. [21] beschrieb für Taillenumfang eine genomweit signifikante Assoziation mit $M C 4 R$, welches uns bereits von den GWAS zu BMI bekannt ist. Unabhängig vom Taillenumfang konnte für dieses Gen auch eine Assoziation mit Insulinresistenz beobachtet werden. In weiteren Untersuchungen von Lindgren et al. wurde neben dem FTO und dem MC4R Gen auch eine Assoziation mit TFAP2B und MSRA beschrieben [22]. In einer weiteren GWAS aus dem CHARGE Konsortium [23] konnten 3 Gene gefunden werden: Die bereits früher gefundenen Gene MC4R [21] und FTO [24] konnten bestätigt werden. Mit NRXN3 konnte ein weiteres sehr interessantes Gen gefunden werden, welches bereits früher in Zusammenhang mit Suchtverhalten gebracht worden war $[25,26]$.

In der oben bereits beschriebenen GWAS zu WHR [20] wurden zusätzlich weitere fünf Phänotypen untersucht: Taillenumfang auf BMI adjustiert, Hüftumfang auf BMI adjustiert, sowie die nicht auf BMI adjustierten Phänotypen Taillenumfang, Hüftumfang und WHR. Diese Analysen haben weitere 19 Genorte identifiziert, von denen 
Abb. 3 Effekte der von Shungin et al. [20] identifizierten 20 Genorte auf WaistHip-Ratio, bei denen in der Effektstärke zwischen Männer und Frauen signifikante Unterschiede bestehen. Die in der Box 1 gezeigten ersten 15 Genorte zeigen nur bei Frauen signifikante Effekte, nicht jedoch bei Männern. In Box 2 finden sich 4 Gene mit einem stärkeren Effekt bei Frauen und einem schwächer ausgeprägten Effekt bei Männern, der in die gleiche Richtung wie bei Frauen geht. In Box 3 findet sich ein Gen mit einem signifikanten Effekt bei Männern, aber keinem Effekt bei Frauen. Daten sind entnommen aus Tab. 1 von Shungin et al. [20]

neun einen stärkeren Effekt bei einem der Geschlechter gezeigt haben ( 4 bei Frauen und fünf bei Männern).

\section{GWAS Ergebnisse bezüglich anderer Phänotypen}

Phänotypen wie BMI, Taillenumfang und WHR sind in zahlreichen Kohortenstudien gemessen worden. Daher war es auch relativ leicht, große Konsortien zu etablieren, um diese Phänotypen in GWAS zu untersuchen. Zu Phänotypen, deren Erhebung aufwändiger ist, gibt es nur wenige GWAS. Hier ist eine Studie von Fox et al. hervorzuheben, die Phänotypen wie subkutanes (SAT), viszerales (VAT) und die VAT/SAT-Ratio in 4 populationsbasierten Studie in zusammen 5560 Frauen und 4997 Männern untersucht hat [27]. Die stärkste Assoziation wurde dabei für das LYPLAL1 Gen mit der VAT/SAT-Ratio gefunden $\left(p=3,1 \times 10^{-09}\right)$, welches früher schon in Zusammenhang mit der WHR beschrieben worden war. Für das FTO-Gen wurde eine signifikante Assoziation mit SAT gefunden $\left(p=5,9 \times 10^{-08}\right)$. Das in Bezug auf geschlechts-spezifische Unterschiede wohl interessanteste Gen wurde für den SNP rs1659258 in der Nähe von THNSL2 gefunden: Hier fand sich eine genomweit signifikante Assoziation mit VAT nur bei Frauen $\left(p=1,6 \times 10^{-08}\right)$, nicht jedoch bei Männern $(p=0,75)$. Diese geschlechtsspezifische Assoziation konnte im GIANT Konsortium für „verwandte“ Phänotypen wie BMI und Taillenumfang bestätigt werden [27]. Die Funktion dieses Gens bedarf erst einer umfassenden Klärung. In einer Nebenuntersuchung konnte für 7 der 14 weiter oben beschriebenen Genorte für WHR [19] und für 7 der oben ebenfalls beschriebenen 32 Genorte für BMI [12] auch eine Assoziation für zumindest einen der Phänotypen SAT, VAT oder VAT/SAT-Ratio beobachtet werden [27].
Neben den direkt mit Adipositas assoziierten Phänotypen sind mittlerweile auch zahlreiche andere intermediäre Phänotypen wie Adiponectin, Leptin u. a. untersucht worden, die in der Pathogenese der Adipositas möglicherweise in wichtige Rolle spielen dürften. Beispielhaft ist hier nur Adiponectin besprochen, welches in Adipozyten gebildet wird und deren Konzentrationen inverse Korrelationen mit klinischen Parametern wie Blutglukose, Insulinresistenz, Dyslipidämie, Typ-2 Diabetes, KHK und Schlaganfall aufweisen [28]. In einer frühen Studie haben wir in der SAPHIR-Studie genetische Varianten im Adiponectin-Gen untersucht und zeigen können, dass mit 7-8\% ein sehr hoher Anteil der Adiponectin-Konzentrationen durch Varianten in diesem Gen erklärbar ist [29]. In nachfolgenden GWAS konnten wir dann in fast 40.000 Studienteilnehmern bis $\mathrm{zu} 12$ Genregionen identifizieren, die einen Einfluss auf die Konzentrationen von Adiponectin haben [30, 31]. Zwei dieser Genregionen (TRIB1 und GPR109) haben bei Frauen einen deutlich stärkeren Effekt als bei Männer gezeigt [31]. Weiters konnten wir zeigen, dass die Summe der Adiponectin-erniedrigenden Allele dieser Genorte auch eine Assoziation mit Typ-2 Diabetes, Triglyzeriden, WHR, Glukose (gemessen nach $2 \mathrm{~h}$ nach Glukosebelastung), Insulin und HDL-Cholesterin zeigen. Dies weist sehr eindrücklich auf die Bedeutung der genetischen Determination dieser Phänotypen über die Adiponectin-Achse hin.

\section{Von der GWAS zur Funktion - ein langer Weg}

Bisher ist von kaum einem der gefundenen Gene aus dem Bereich der Adipositas die Funktion im Detail aufgeklärt worden und es wird auch noch sehr lange dauern, bis diese Funktionsweisen durchleuchtet sein werden. Für 
FTO (fat mass and obesity associated protein), welches die stärkste Assoziation zu Adipositas zeigt, ist dies kürzlich sehr eindrucksvoll gelungen [32]. Liegt ein C-Allel in SNP rs1421085 vor, wird das ARID5B Repressor-Motiv zerstört. Dies führt wiederum zu Derepression eines potenten Preadipozyten-Enhancers und einer massiven Verstärkung der Expression von IRX3 und IRX5 während der frühen Adipozyten-Differenzierung. Dadurch kommt es in weiterer Folge zu einer Umstellung der Zellentwicklung von energieverbrauchenden braunen Fettzellen zu energiespeichernden weißen Fettzellen. Dies führt zu einer Reduktion der mitochondrialen Thermogenese um den Faktor 5 und einem Anstieg der Lipideinlagerung [32].

Ein möglicher anderer Mechanismus, wie genetische Varianten Adipositas beeinflussen können, ist der epigenetische Regulationsmechanismus. Darunter versteht man die vererbbaren, aber durchaus reversiblen Veränderungen der Genexpression, wobei die DNASequenz dabei grundsätzlich nicht verändert ist. Die bisher wichtigsten bekannten Mechanismen sind DNAMethylierung, Histonmodifikation, Verpackung der DNA um Nukleosomen und Chromatinfaltung. Welche Rolle spielen SNPs in diesen Mechanismen? Es konnte bereits gezeigt werden, dass selbst SNPs in großer Distanz, sogar auf anderen Chromosomen, mit Veränderungen der DNA-Methylierung und damit der Regulation von Genen assoziiert sind [33]. Für Adipositas konnte ein solcher Zusammenhang mit DNA-Methylierung für 28 SNPs gezeigt werden, die ursprünglich in GWAs identifiziert wurden [34]. Die systematischen Untersuchungen des gesamten „Epigenoms“ stecken momentan noch in den Anfängen. Es kann aber angenommen werden, dass einige der in GWAS identifizierten Assoziationen auch durch epigenetische Regulationsmechanismen erklärt werden können.

\section{Was haben wir aus den besprochenen Studien gelernt?}

- Die Einführung der genomweiten Assoziationsstudien (GWAS) hat in den letzten 5-7 Jahren zu einem sprunghaften Anstieg der Anzahl der Gene geführt, die mit Adipositas und damit zusammenhängender Phänotypen wie BMI, Taillenumfang und Waist-HipRatio assoziiert gefunden worden sind.

- Jedes einzelne dieser Gene erklärt nur einen sehr kleinen Anteil der Varianz dieser Phänotypen. Dies bedeutet allerdings keinesfalls, dass diese Gene nur eine untergeordnete Rolle spielen.

- Geschlechtsspezifische Unterschiede kennen wir hauptsächlich nur für den Phänotyp Waist-Hip-Ratio. Ca. zwei Fünftel für diesen Phänotyp bekannten Genvarianten weist bei Frauen einen deutlich stärkeren Effekt wie bei Männern auf.

- Die funktionelle Charakterisierung der identifizierten Gene und Genvarianten und das Verständ- nis der Pathogenese wird noch lange Zeit in Anspruch nehmen.

- Gene kennen keine Grenzen: Die multinationale Zusammenarbeit in verschiedenen Konsortien hat es überhaupt erst möglich gemacht, dass wir heute diese hohe Anzahl an Genen für die genannten Phänotypen kennen.

- Ob sich aus den gefundenen Ergebnissen in Zusammenhang mit Adipositas jemals therapeutische Konsequenzen ergeben, lässt sich heute schwer abschätzen. Das Verständnis der involvierten Regelkreise ist aber unabdingbare Voraussetzung für die Entwicklung neuer Therapien.

Die Suche nach Genen, die für Übergewicht prädisponieren, ist erst am Anfang. Der Großteil des geschätzten vererbbaren Anteils von Übergewicht ist noch ungeklärt. Weitere Zusammenhänge werden in seltenen genetischen Varianten vermutet, die nicht in GWAS untersucht werden können, in bisher nicht berücksichtigten Gen-Umwelt-Interaktionen oder auch in epigenetischen Regulationsmechanismen.

\section{Einhaltung ethischer Richtlinien}

\section{Interessenkonflikt}

Claudia Lamina, Bernhard Paulweber und Florian Kronenberg geben an, dass kein Interessenkonflikt besteht.

\section{Open Access}

Dieser Artikel unterliegt den Bedingungen der Creative Commons Attribution License. Dadurch sind die Nutzung, Verteilung, und Reproduktion erlaubt, sofern der/die Originalautor/en und die Quelle angegeben sind.

\section{Literatur}

1. Neel JV. Diabetes mellitus: a "thrifty" genotype rendered detrimental by „progress“? Am J Hum Genet. 1962;14:353-62.

2. Speakman JR. Thrifty genes for obesity, an attractive but flawed idea, and an alternative perspective: the ,drifty gene' hypothesis. Int J Obes (Lond). 2008;32:1611-7.

3. Bouchard C. The biological predisposition to obesity: beyond the thrifty genotype scenario. Int J Obes (Lond). 2007;31:1337-9.

4. Wells JC. The evolution of human adiposity and obesity: where did it all go wrong? Dis Model Mech. 2012;5:595-607.

5. Stunkard AJ, Foch TT, Hrubec Z. A twin study of human obesity. JAMA. 1986;256:51-4.

6. Haworth CM, Carnell S, Meaburn EL, et al. Increasing heritability of BMI and stronger associations with the FTO gene over childhood. Obesity (Silver Spring). 2008;16:2663-8.

7. Hjelmborg J, Fagnani C, Silventoinen K, et al. Genetic influences on growth traits of BMI: a longitudinal study of adult twins. Obesity (Silver Spring). 2008;16:847-52.

8. Farooqi IS, O'Rahilly S. Monogenic obesity in humans. Annu Rev Med. 2005;56:443-58.

9. Xia Q, Grant SF. The genetics of human obesity. Ann N Y Acad Sci. 2013;1281:178-90. 
10. Visscher PM, Brown MA, McCarthy MI, et al. Five years of GWAS discovery. Am J Hum Genet. 2012;90:7-24.

11. Kronenberg F. Genome-wide association studies in agingrelated processes such as diabetes mellitus, atherosclerosis and cancer. Exp Gerontol. 2008;43:39-43.

12. Speliotes EK, Willer CJ, Berndt SI, et al. Association analyses of 249,796 individuals reveal 18 new loci associated with body mass index. Nat Genet. 2010;42:937-48.

13. Locke AE, Kahali B, Berndt SI, et al. Genetic studies of body mass index yield new insights for obesity biology. Nature. 2015;518:197-206.

14. Guh DP, Zhang W, Bansback N, et al. The incidence of comorbidities related to obesity and overweight: a systematic review and meta-analysis. BMC Public Health. 2009;9:88.

15. Castro AV, Kolka CM, Kim SP, et al. Obesity, insulin resistance and comorbidities? Mechanisms of association. Arq Bras Endocrinol Metabol. 2014;58:600-9.

16. Tchernof A, Despres JP. Pathophysiology of human visceral obesity: an update. Physiol Rev. 2013;93:359-404.

17. Canoy D. Distribution of body fat and risk of coronary heart disease in men and women. Curr Opin Cardiol. 2008;23:591-8.

18. Wang Y, Rimm EB, Stampfer MJ, et al. Comparison of abdominal adiposity and overall obesity in predicting risk of type 2 diabetes among men. Am J Clin Nutr. 2005;81:555-63.

19. Heid IM, Jackson AU, Randall JC, et al. Meta-analysis identifies 13 new loci associated with waist-hip ratio and reveals sexual dimorphism in the genetic basis of fat distribution. Nat Genet. 2010;42:949-62.

20. Shungin D, Winkler TW, Croteau-Chonka DC, et al. New genetic loci link adipose and insulin biology to body fat distribution. Nature. 2015;518:187-96.

21. Chambers JC, Elliott P, Zabaneh D, et al. Common genetic variation near MC4R is associated with waist circumference and insulin resistance. Nat Genet. 2008;40:716-8.

22. Lindgren CM, Heid IM, Randall JC, et al. Genome-wide association scan meta-analysis identifies three loci influencing adiposity and fat distribution. PLoS Genet. 2009;5:e1000508.
23. Heard-Costa NL, Zillikens MC, Monda KL, et al. NRXN3 is a novel locus for waist circumference: a genome-wide association study from the CHARGE Consortium. PLoS Genet. 2009;5:e1000539.

24. Kring SI, Holst C, Zimmermann E, et al. FTO gene associated fatness in relation to body fat distribution and metabolic traits throughout a broad range of fatness. PLoS One. 2008;3:e2958.

25. Lachman HM, Fann CS, Bartzis M, et al. Genomewide suggestive linkage of opioid dependence to chromosome 14q. Hum Mol Genet. 2007;16:1327-34.

26. Clay SW, Allen J, Parran T. A review of addiction. Postgrad Med. 2008;120:E01-7.

27. Fox CS, Liu Y, White CC, et al. Genome-wide association for abdominal subcutaneous and visceral adipose reveals a novel locus for visceral fat in women. PLoS Genet. 2012;8:e1002695.

28. Tilg $\mathrm{H}$, Moschen AR. Adipocytokines: mediators linking adipose tissue, inflammation and immunity. Nat Rev Immunol. 2006;6:772-83.

29. Heid IM, Wagner SA, Gohlke H, et al. Genetic architecture of the $A P M 1$ gene and its influence on adiponectin plasma levels and parameters of the metabolic syndrome in 1727 healthy Caucasians. Diabetes. 2006;55:375-84.

30. Heid IM, Henneman P, Hicks A, et al. Clear detection of ADIPOQ locus as the major gene for plasma adiponectin: results of genome-wide association analyses including 4659 European individuals. Atherosclerosis. 2010;208:412-20.

31. Dastani Z, Hivert MF, Timpson N, et al. Novel Loci for adiponectin levels and their influence on type 2 diabetes and metabolic traits: a multi-ethnic meta-analysis of 45,891 individuals. PLoS Genet. 2012;8:e1002607.

32. Claussnitzer M, Dankel SN, Kim KH, et al. FTO obesity variant circuitry and adipocyte browning in humans. $\mathrm{N}$ Engl J Med. 2015;373:895-907.

33. Lemire M, Zaidi SH, Ban M, et al. Long-range epigenetic regulation is conferred by genetic variation located at thousands of independent loci. Nat Commun. 2015;6:6326.

34. Voisin S, Almen MS, Zheleznyakova GY, et al. Many obesityassociated SNPs strongly associate with DNA methylation changes at proximal promoters and enhancers. Genome Med. 2015;7:103. 